\title{
ON AN EXTENDED HADAMARD MAXIMUM DETERMINANT PROBLEM
}

\author{
D. JOIŢA AND L. NĂSTĂSESCU
}

Abstract. Motivated by the Hadamard maximum determinant problem we study the quantities $a_{m, n}=\max \operatorname{det}\left(A A^{T}\right)$ where $A$ is a $m \times n$ matrix with entries 1 and -1 . We find the exact values of $a_{2, n}$ and $a_{3, n}$ and for a general $m$ we give upper and lower bounds for $a_{m, n}$.

Mathematics subject classification (2010): 15A15, 15A36.

Keywords and phrases: \pm 1 matrices, maximum determinant problem.

\section{REFERENCES}

[1] J. Brenner, L. Cummings, The Hadamard maximum determinant problem, Amer. Math. Monthly, 79 (1972), 626-630.

[2] J. H. E. CoHn, On the value of determinants, Proc. Amer. Math. Soc., 14 (1963), 581-588.

[3] H. EHLICH, Determinantenabschätzungen für binäre Matrizen, Math. Z., 83 (1964), 123-132.

[4] H. EHLICH, Determinantenabschätzung für binäre Matrizen mit $n \equiv 3 \bmod 4$, Math. Z., 84 (1964), $438-447$.

[5] D. K. Faddeev, I. S. Somins Kil, Problems in higher algebra, Freeman and Co., San FranciscoLondon, 1965.

[6] M. G. Neubauer, A. J. Radcliffe, The maximum determinant of \pm 1 matrices, Linear Algebra Appl., 257 (1997), 289-306. 